\title{
INFLUÊNCIA DE ERROS CONSTRUTIVOS NO DESEMPENHO ACÚSTICO DE PAREDES DE ALVENARIA
}

\author{
H. SANTOS LABRES \\ Engenheiro Civil \\ Prefeitura Municipal de Taquari \\ Rio Grande do Sul; Brasil \\ hslabres@gmail.com
}

\section{F. PACHECO}

Engenheira civil

itt Performance - Unisinos

Rio Grande do Sul; Brasil

fernandapache@unisinos.br

\section{B. FONSECA TUTIKIAN}

Engenheiro civil

itt Performance - Unisinos

Rio Grande do Sul; Brasil

bftutikian@unisinos.br

\author{
M F. DE OLIVEIRA \\ Arquiteta e urbanista \\ itt Performance - Unisinos \\ Rio Grande do Sul; Brasil \\ mariaon@unisinos.br \\ M J. OTT \\ Engenheira civil \\ itt Performance - Unisinos \\ Rio Grande do Sul; Brasil \\ mairajo@unisinos.br
}

\section{RESUMO}

Defeitos de construção surgidos na etapa de execução, quando pode existir dificuldade de controle, mesmo que visualmente não rastreáveis, podem comprometer o desempenho acústico dos sistemas construtivos. Este artigo tem como objetivo avaliar a influência de erros construtivos no isolamento de sons aéreos. Para isso, foram construídas amostras de paredes de alvenaria com defeitos induzidos, relacionados a técnicas construtivas (juntas e encunhamento) e sistemas complementares (instalações). Em comparação com a amostra referência, foram encontradas reduções de $1 \mathrm{~dB}$ para as amostras com falta de preenchimento adequado do encunhamento, e $2 \mathrm{~dB}$ para as amostras sem preenchimento das juntas verticais. Em relação às amostras com instalações, essa redução atingiu 1 e $3 \mathrm{~dB}$ para amostras com instalação de caixas de junções elétricas na mesma face e duas em cada face, respectivamente. Percebe-se assim que não apenas a especificação de projeto, mas também a análise de conformidade da construção exercem influência no desempenho acústico.

Palavras-chave: desempenho acústico, paredes de alvenaria, erros construtivos, instalações.

\section{ABSTRACT}

Construction defects arisen on the execution phase were is hard to control these, even visually untraceable, can forfeit the acoustic performance of systems. This article aims to evaluate the influence of these construction defects in front of airborne sound insulation. Wall system samples of real proportions were made with induced defects, related to construction techniques (joints and gaps above the wall) and complementary systems (installations). In comparison with the reference, reductions of $1 \mathrm{~dB}$ were found for the samples with defective gaps above the wall and $2 \mathrm{~dB}$ for the trials with no vertical filling. Regarding the samples with installations, this reduction reached 1 and $3 \mathrm{~dB}$ for samples with installation of electrical junction boxes at the same face and two on each face, respectively. It is noticed that not only the project specification, but also a construction conformity analysis influences the acoustic performance.

Keywords: acoustic performance, brick masonry walls, construction defects, installations.

\section{INTRODUÇÃO}

Os princípios do isolamento acústico a sons aéreos de sistemas construtivos pesados consideram o aumento da perda de transmissão sonora de acordo com o aumento da massa superficial, com base na Lei da Massa, ou conforme o princípio massa-mola-massa para sistemas construtivos leves. (BALLOU, 2008). Embora seja possível prever possíveis fontes de ruídos em um edifício e escolher materiais de construção com isolamento acústico eficiente, o desempenho acústico 
satisfatório de um sistema construtivo pode não ser totalmente garantido, dada a forte dependência com a execução correta do edifício. (MATEUS e PEREIRA, 2011).

Craik e Evans (1989) afirmaram que o isolamento acústico de sons aéreos de sistemas construtivos, com especificações idênticas, ainda pode mostrar diferenças de cerca de $2 \mathrm{~dB}$ para o índice de redução sonora ponderado (Rw). Segundo os autores, essas diferenças não podem ser explicadas pelas propriedades dos materiais ou pelas transmissões por flancos, mas pela diferença no processo de execução da própria obra.

Barrett (2009) usa o conceito estabelecido por Tate Latham e Son para explicar o significado do defeito, que é "uma falta ou ausência de algo essencial à perfeição", mas não há uma maneira única de expressar o que essas imperfeições representam. Geralmente, o conceito de defeito não tem apenas um significado, mas vários, com o objetivo de apontar não-conformidades, erros, falhas e outros. O defeito em si pode ser designado como resultado de um erro, imperfeição ou falha que, na construção civil, está vinculado às várias etapas dos processos de construção.

Fausti et al. (2010) apontam a existência de uma ampla variedade de resultados para medições acústicas, com base na incerteza de medição, condições de teste, fonte de materiais e outros. No entanto, os efeitos dos erros construtivos podem comprometer os resultados, gerando diferenças muito maiores que as esperadas. Não apenas os erros relacionados à execução podem impactar no desempenho acústico, mas também a falta de detalhes no projeto e a má interpretação do executor.

Os sistemas construtivos são sempre propensos a um melhor desempenho acústico quando testados em laboratório e não em campo, devido à possibilidade da transmissão sonora por flancos e a ocorrência de imperfeições durante a construção do edifício. Segundo Peters et al. (2013), essa é a razão pela qual os projetistas têm em seus projetos considerações de isolamento acústico do conjunto de sistemas e elementos construtivos inferiores aos valores declarados em testes de laboratório do sistema isoladamente, ao calcular a perda de transmissão sonora. No entanto, o valor a ser reduzido depende de situações particulares e da experiência do projetista, que, segundo o autor, pode ser de até $8 \mathrm{~dB}$.

Isso implica na necessidade de definir as melhores soluções técnicas a serem aplicadas, desenvolvendo projetos detalhados e de fácil compreensão, além de procedimentos efícientes de controle na etapa de construção. Hopkins (2012), porém, considera que os elementos e sistemas construtivos indicados originalmente na etapa de projeto são frequentemente substituídos na fase de construção e que a qualidade do acabamento pode variar muito dentro do mesmo edifício.

As estimativas teóricas de isolamento acústico consideram que os sistemas construtivos homogêneos e isotrópicos, embora a maioria dos elementos de construção seja feita de pequenos componentes, como tijolos que formam uma parede de alvenaria. Na prática, as propriedades dos elementos sempre apresentarão alguma variação em relação à perda de transmissão sonora, devido não apenas às características dos materiais e suas conexões, mas também às variações em sua forma de instalação (HOPKINS, 2012). Essas variações fazem com que os materiais, elementos e sistemas construtivos se caracterizem como não homogêneos, principalmente em sistemas que requerem a inclusão de instalações como, por exemplo, caixas elétricas ou tubulações. Nessas situações, os vazios e alterações no meio de propagação podem tornar as as estimativas teóricas muito imprecisas. (DEMIRKALE e ASCIGIL-DINCER, 2017).

Carrascal et al. (2014) listam algumas das causas mais frequentes de reclamações de usuários de edificações, que combinam emissão constante de ruído e baixo isolamento dos sistemas construtivos que limita os espaços internos. Entre os espaços internos mais problemáticos citados, estão os relacionados à produção de ruído, como banheiros, cozinhas e escadas, interconectados a quartos e salas de estar. Câmaras de tubulação hidráulica, salas de máquinas e garagens próximas a zonas silenciosas também são mencionadas, e as soluções relacionadas a essas considerações devem ser definidas durante a fase de projeto. Portanto, o projeto das paredes que separam os ambientes deve considerar dois aspectos fundamentais, que são: (1) escolha de uma partição que atenda aos requisitos normativos, e (2) desenvolvimento de detalhes construtivos das conexões, para minimizar a transmissão por flancos.

Considerando a grande influência das variações na eficiência do isolamento acústico dos sistemas construtivos, estudos recentes sobre o desempenho acústico com base em testes de campo, tiveram um controle rigoroso de todo o processo de construção da amostra incluído em seus métodos. (DEL VAL L et al., 2018). Carrascal et al. (2014) dão exemplos de erros comuns identificados em vários países europeus, que comprometem o isolamento acústico aéreo dos sistemas de parede. Alguns dos principais estão relacionados à falta de argamassa nas juntas verticais entre os blocos, levando a numerosos 
espaços vazios que aumentam a transmissão do som entre as salas e aos espaços entre o topo da parede de alvenaria e a laje.

Mateus e Pereira (2012) estudaram a influência do preenchimento do encunhamento em uma parede dupla de alvenaria de blocos cerâmicos, com espessura de parede de $11 \mathrm{~cm}+11 \mathrm{~cm}$, com camada de ar, com diferentes tipos de acabamento. Os resultados dos ensaios em campo indicaram um aumento de $2 \mathrm{~dB}$ na diferença de nível padronizada ponderada quando o encunhamento foi totalmente preenchido.

A alvenaria é um sistema de construção amplamente utilizado. No entanto, pode ser percebida uma certa dificuldade pelos profissionais que executam esse tipo de fechamento, o que é visível a partir da constatação de defeitos construtivos que aparecem durante o processo de execução das alvenarias. Como explica Ioshimoto (1988), a ocorrência de problemas ou manifestações patológicas pode ter origem em quaisquer das etapas de construção ou de utilização das edificações, sendo relacionado diretamente ao nível de controle de qualidade exercido em cada uma dessas etapas. (NICHELE, 2014; MESOMO, 2018).

Segundo Sousa e Cabral (2014), os erros mais comuns que ocorrem durante a execução de alvenaria de blocos são a perda de prumo, uso de blocos quebrados, o não preenchimento ou preenchimento ineficaz de juntas e a falta de uniformidade quanto à a espessura dessas articulações. Outros problemas mencionados pelos autores que podem comprometer a qualidade da alvenaria de tijolos incluem rejuntes mal executados e rasgos na alvenaria para a passagem de dutos para a fiação elétrica.

A proposta deste artigo baseia-se na necessidade de quantificar o impacto das variações nas maneiras de construir elementos de um edifício. Portanto, objetiva avaliar a influência de defeitos induzidos em paredes de alvenaria no desempenho acústico.

\section{MATERIAIS E MÉTODOS}

\subsection{Planejamento do programa experimental}

O programa experimental constitui-se em definir os sistemas de construção a serem avaliados, seus detalhes de construção e / ou modificações a serem aplicadas, a fim de distinguir as amostras de teste das amostras de referência. Em seguida, foi realizada a caracterização dos materiais utilizados durante a execução da alvenaria de cada amostra, como argamassas de assentamento e revestimento de blocos cerâmicos. Após, os testes foram realizados e os resultados, analisados.

\subsection{Definição dos sistemas de construção}

Por razões de comparação, considerou-se a construção de uma parede de referência, ou seja, a execução da parede que seguiu rigorosamente as técnicas construtivas, com um controle preciso do prumo e preenchimento total de juntas verticais e horizontais, ambas com espessura média de $1 \mathrm{~cm}$. As demais amostras utilizadas neste estudo apresentavam algum tipo de defeito construtivo, como será especificado a seguir.

$\mathrm{Na}$ amostra com falha no preenchimento do encunhamento, o defeito induzido foi localizado na superfície de contato entre a última fila de tijolos e a viga do pórtico de teste. A saber, essa parede foi executada de maneira idêntica à amostra de referência, exceto pelo espaço vazio de $3 \mathrm{~cm}$ entre a última fila de tijolos e o pórtico de teste ao longo do comprimento da parede.

A outra amostra foi construída com defeitos induzidos nas juntas verticais de assentamento dos blocos, de maneira que não fossem preenchidas, mantendo-se o espaçamento de $1 \mathrm{~cm}$.

A última parede construída tinha as mesmas características da amostra de referência e nela foram inseridas caixas de derivação elétrica. Primeiramente, foram colocadas duas caixas de derivação elétrica de $10 \times 5 \mathrm{~cm}$, instaladas em uma de suas faces, espaçadas a 1,60 m, nas quais foi colocada uma placa de saída dupla. Em seguida, duas caixas de junção elétrica adicionais foram instaladas alinhadas com as já existentes, na outra face da parede.

A Tabela 1 apresenta a identificação dos sistemas construtivos e suas características gerais. Cada sistema teve uma massa superficial aproximada de $200 \mathrm{~kg} / \mathrm{m}^{2}$, enquanto que a amostra $\mathrm{S} 1$ teve uma redução inferior a $3 \mathrm{~kg} / \mathrm{m}^{2}$. A espessura total 
das amostras foi de $22 \mathrm{~cm}$, confeccionadas em alvenaria de blocos cerâmicos de $19 \times 19 \times 29$ cm, que receberam revestimento de argamassa de $2 \mathrm{~cm}$ na face externa e $1 \mathrm{~cm}$ na face interna. A argamassa utilizada durante a execução das paredes, para assentamento e revestimento, foi fornecida em estado pronto para uso, com fluidez estável. A execução das amostras durou três dias consecutivos, dos quais dois foram utilizados para a execução da alvenaria e chapisco com agregado graúdo de dimensão superior a $5 \mathrm{~mm}$, um para o revestimento de $1 \mathrm{~cm}$ da face interna, e o outro para o revestimento de $2 \mathrm{~cm}$ da face externa. Após, houve um período de espera de 7 dias para a secagem as amostras fora da câmara de teste. Esse período de 7 dias foi validado em estudo anterior de Klippel et al. (2018) para ensaios de perda de transmissão sonora em sisteamas de vedação vertical com revestimento argamassado.

Tabela 1 - Identificação das amostras ensaiadas

\begin{tabular}{ll}
\hline Identificação do sistema & Descrição \\
\hline REF & $\begin{array}{l}\text { Alvenaria de bloco cerâmico com } \\
\text { juntas de } 1 \mathrm{~cm} .\end{array}$ \\
S1 & $\begin{array}{l}\text { Alvenaria de bloco cerâmico com } \\
\text { defeito induzido no encunhamento. }\end{array}$
\end{tabular}

S2

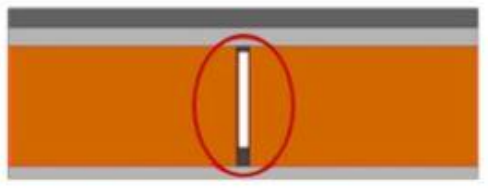

S3

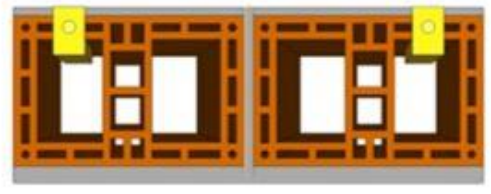

S4

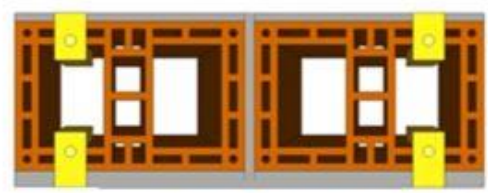

Alvenaria de bloco cerâmico com defeito induzido nas juntas de assentamento.

\section{Alvenaria de bloco cerâmico com duas caixas de junção elétrica $10 \times 5$ $\mathrm{cm}$ instaladas na mesma face, espaçadas a $1,60 \mathrm{~m}$.}

\author{
Alvenaria de bloco cerâmico com \\ quatro caixas de junção elétrica \\ $10 \times 5 \mathrm{~cm}$ instaladas, duas em cada \\ face, espaçadas a $1,60 \mathrm{~m}$.
}

\subsection{Caracterização dos materiais}

Para caracterizar os blocos foi utilizada uma amostragem de 13 blocos, que foram submetidos à determinação de suas características geométricas (Figura 1 (a)), índice de absorção de água e resistência axial à compressão (Figura 1 (b)). Os testes nos blocos foram realizados de acordo com a norma ASTM C67-1713 no Laboratório de Materiais de Construção da Unisinos. De acordo com as medições realizadas, os desvios de esquadro variaram de 1,11 a 2,90 mm. As argamassas foram caracterizadas no estado fresco e endurecido através da determinação da densidade de massa, conforme EN 1015 6:2014 e EN 1015-10:2015.

Os ensaios citados para caracterizar os materiais utilizados foram realizados com o objetivo de certificar que estes atendem aos requisitos das normas e assim, garantir as mesmas condições para a construção de todas as amostras e permitir a reprodução dos ensaios por outro pesquisador (Tabela 2). 

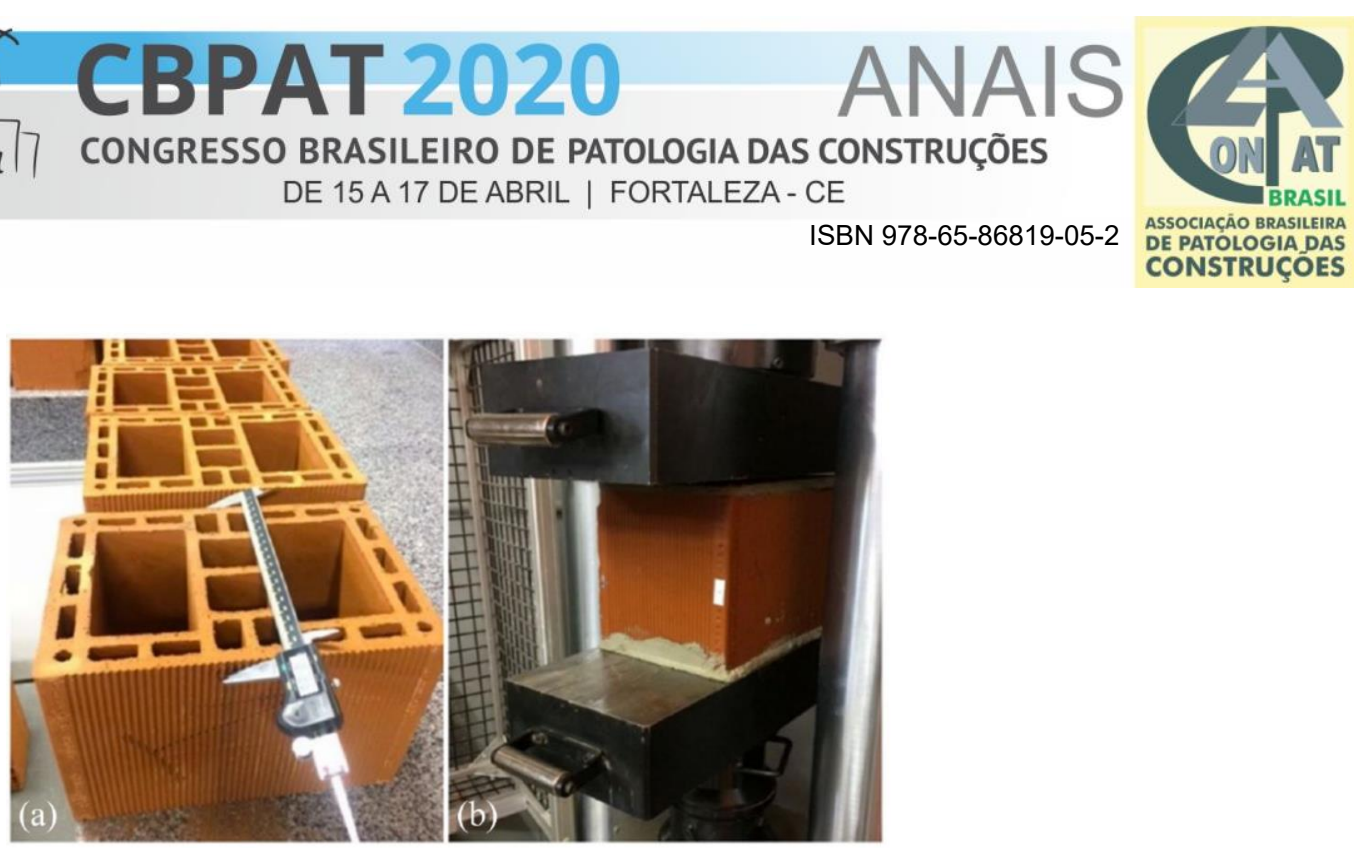

Figura 1 - Caracterização de blocos cerâmicos: (a) características geométricas e (b) teste de resistência à compressão axial

Tabela 2 - Resultados da caracterização dos materiais

\begin{tabular}{|c|c|c|c|}
\hline \multicolumn{2}{|c|}{ Argamassa } & \multicolumn{2}{|c|}{ Blocos cerâmicos } \\
\hline $\begin{array}{l}\text { Densidade de massa } \\
\text { aparente da argamassa no } \\
\text { estado fresco }\left(\mathrm{kg} / \mathrm{m}^{3}\right)\end{array}$ & $\begin{array}{l}\text { Densidade de massa aparente } \\
\text { no estado endurecido da } \\
\text { argamassa }\left(\mathrm{kg} / \mathrm{m}^{3}\right)\end{array}$ & Absorção de água (\%) & $\begin{array}{l}\text { Resistência axial à } \\
\text { compressão }\end{array}$ \\
\hline 1834,65 & 1680,00 & 18,16 & 4,90 \\
\hline
\end{tabular}

\subsection{Ensaios de isolamento sonoro}

As câmaras de reverberação acústica utilizadas para os ensaios deste estudo estão localizadas no Laboratório de Desempenho Acústico do Instituto Tecnológico de Desempenho e Construção Civil (itt Performance) da Unisinos (Figura 2 (a)). A sala de emissão e recepção (Figura 2 (d)) não é unida e as amostras são construídas dentro de molduras que se movem sobre trilhos metálicos colocados no chão e alinhados ao teto (Figura 2 (c)). Um sistema pneumático feito de câmaras-de-ar elastoméricas e ar comprimido garante a vedação, sem adulterar o resultado por transmissão de flanco, entre as duas salas (Figura 2 (b)), e o manuseio das amostras é possível devido às alças com cabos de aço que estão ancoradas na estrutura, o que permite içar as amostras com um guindaste de ponte (Figura 2 (c)). 


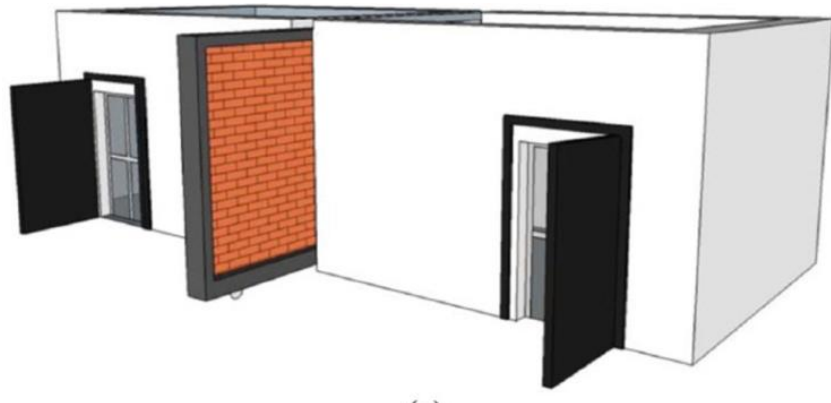

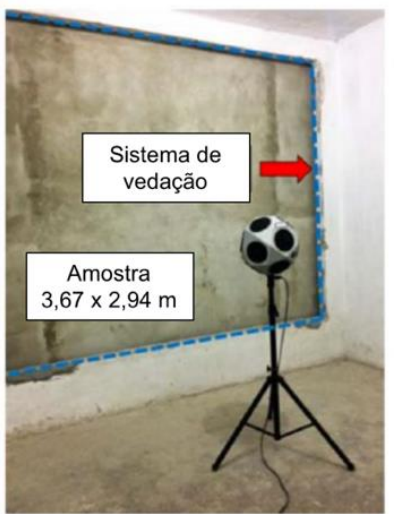

(b)

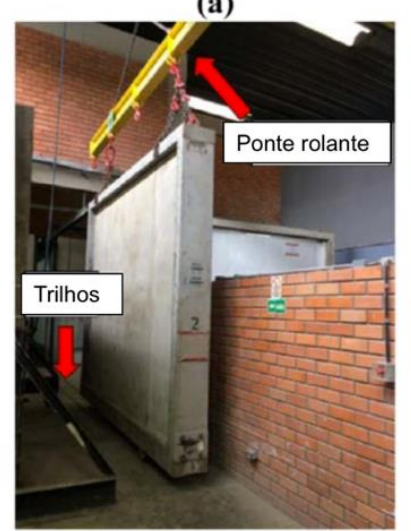

(c)

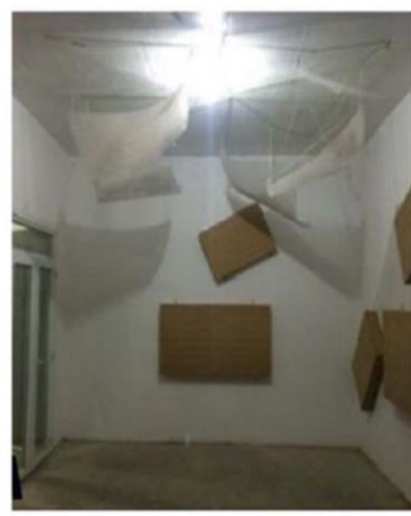

(d)

Figura 2 - Câmaras de reverberação para ensaios de isolamento acústico: (a) visão geral de salas e amostras, (b) sala de emissão, (c) sistema para movimentação de amostras e (d) sala de recepção

Os testes de laboratório foram realizados conforme prescrito pelas normas ISO 10140-2 e ISO 10140-4, que especificam o método para medir o isolamento acústico quanto ao ruído aéreo de produtos de construção, como paredes, janelas, portas e outros, a fim de obter o índice de redução sonora ponderado Rw. Todas as medidas foram realizadas em bandas de terço de oitava, considerando as frequências centrais de 100 a $5000 \mathrm{~Hz}$. Foram utilizados equipamentos Brüel \& Kjær, sendo o microfone tipo 4189, pré-amplificador tipo ZC-0032, analisador portátil tipo 2270, calibrador de som tipo 4231, amplificador de potência tipo 2734-A e alto-falante omnidirecional de alta potência tipo 4292L. A temperatura e a umidade relativa do ar foram monitoradas durante os testes, dentro e fora de cada sala, para que os testes pudessem ser realizados em condições de temperatura acima de $15{ }^{\circ} \mathrm{C}$ e umidade relativa do ar no intervalo de $30 \%$ a $90 \%$, conforme prescreve a ISO 10140-2.

\section{ANÁLISE E DISCUSSÃO DOS RESULTADOS}

\subsection{Defeitos induzidos por lacunas acima da parede}

A Figura 3 apresenta uma comparação entre os resultados da amostra de referência e da amostra S1, com falha no preenchimento do encunhamento, induzida como defeito construtivo. 


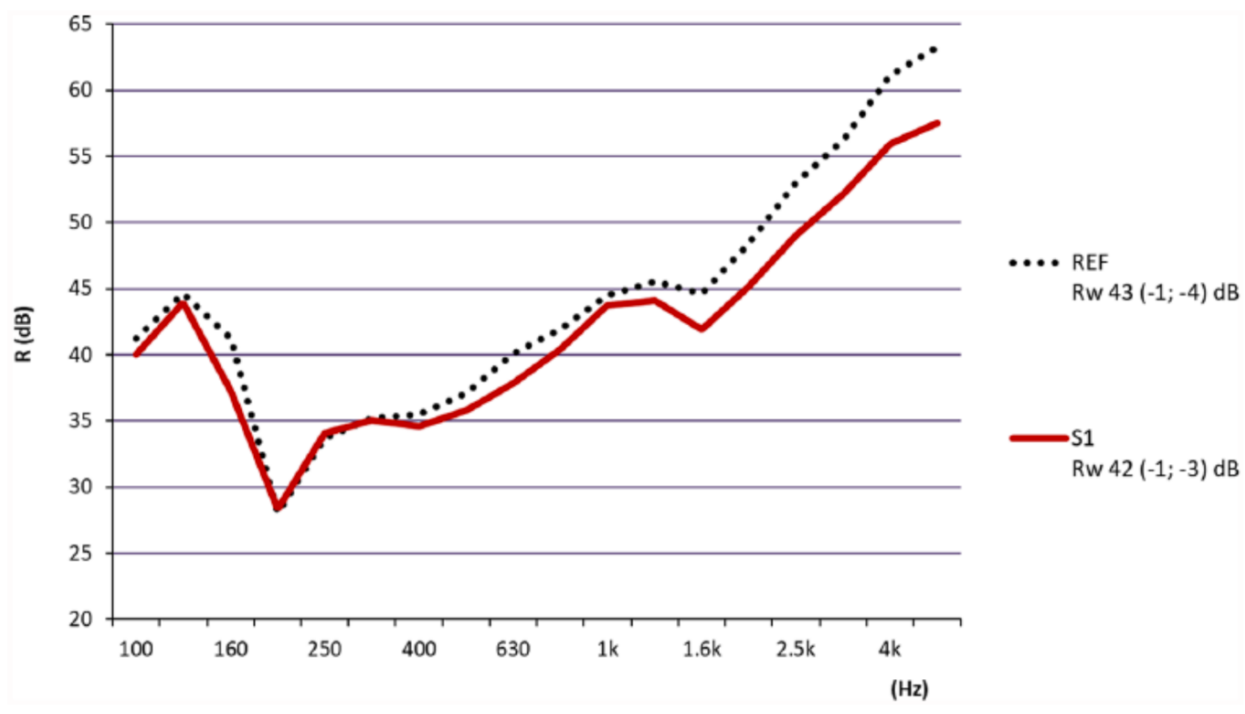

Figura 3 - Comparação entre os resultados da parede de referência REF e a amostra S1

Os resultados dessa parede, comparados com os resultados da amostra de referência, mostraram algumas diferenças até a faixa de frequência de $1000 \mathrm{~Hz}$. Essas variações representam uma perda de isolamento entre as faixas de frequência de 100 a $160 \mathrm{~Hz}$ e 400 a $800 \mathrm{~Hz}$. Consequentemente, a maior diferença em termos de transmissão sonora ocorreu nas bandas de altas frequências, especialmente naquelas acima de $3150 \mathrm{~Hz}$, devido aos menores comprimentos de onda e suas relações em relação aos intervalos existentes de aproximadamente $3 \mathrm{~cm}$ (neste caso). Essa conclusão também pode ser alcançada em estudos realizados por Gomperts, Kihlman e Jones (1967).

Além disso, a região afetada pela ressonância mostrou uma redução expressiva no isolamento entre as bandas de frequência de 160 e $200 \mathrm{~Hz}$ para a amostra de referência, identificada como sendo a vibração do painel com pouco amortecimento, o que aumentou na perda de isolamento. Fringuellino e Smith (1999) investigaram paredes de blocos vazados e identificaram uma dificuldade de previsão de quedas no isolamento provocadas pela ressonância por causa, porque as alvenarias apresentam um comportamento tipicamente ortotrópico. No estudo apresentado neste trabalho, a amostra com falha no preenchimento do encunhamento, S1, apresentou um comportamento semelhante. Assim, a fragilidade induzida por esse defeito de preenchimento não resultou em alterações na frequência ressonante do sistema, a qual está relacionada à vibração da região central. Portanto, a frequência de coincidência de ambos os sistemas ocorreu entre as bandas de frequência de 1250 e $1600 \mathrm{~Hz}$, com subsequente subida da curva ascendente, embora com menores perdas de transmissão para a amostra defeituosa. A perda de isolamento foi de cerca de $4 \mathrm{~dB}$ para frequências acima da faixa de frequência de $1600 \mathrm{~Hz}$.

\subsection{Amostras sem enchimento da junta vertical}

A Figura 4 mostra os resultados da amostra cujas juntas verticais entre os blocos não foram preenchidas (S2). Esses resultados indicam que os vazios modificaram o comportamento da curva de isolamento da parede, incluindo regiões típicas como a controlada pela rigidez, que sofreu a maior perda entre 160 e $200 \mathrm{~Hz}$, em comparação com a parede de referência. Esse sistema apresentou uma transmissão sonora maior para frequências abaixo de $160 \mathrm{~Hz}$ e acima de $250 \mathrm{~Hz}$. O aumento do isolamento acústico nas faixas de frequência de $160 \mathrm{a} 250 \mathrm{~Hz}$, quando comparado à amostra de referência, pode ser explicado com base em um efeito combinado de absorção de energia nas fendas periódicas, devido ao não preenchimento das juntas verticais da parede de alvenaria S2. Estes efeitos combinados também foram observados por Wang (2010) e Sugie, Yoshimura e Iwase (2009). 


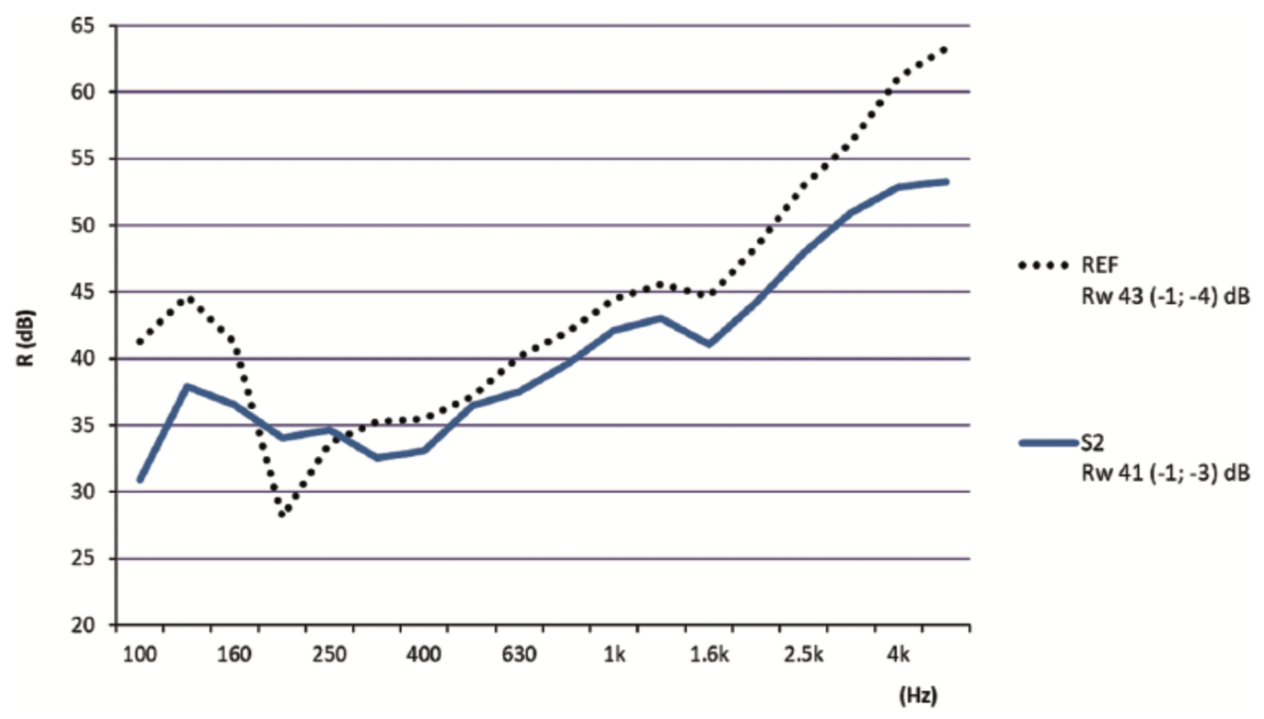

Figura 4 - Comparação entre os resultados da parede de referência REF e amostra S2

Os sistemas que apresentam vazios sequenciais resultaram em padrões de isolamento difíceis de prever, com maior vulnerabilidade em altas frequências devido à possibilidade de livre passagem de sons com comprimentos de onda menores. Hopkins (2012) observou esse mesmo efeito como um problema frequente em alguns edifícios, que apresentam sistemas com características acústica semelhantes à uma placa porosa. Nesse sentido, a curva da amostra S2 se comportou de maneira ligeiramente semelhante em relação à parede referência, nas frequências acima de $500 \mathrm{~Hz}$, mas as perdas de isolamento foram intensificadas à medida que a frequência aumentou. A diferença do índice de redução sonora ponderado foi de $2 \mathrm{~dB}$ para S2, revelando que o aumento na espessura do revestimento reduziu drasticamente o impacto no resultado ponderado, embora defeitos de construção sejam facilmente detectados, de acordo com as mudanças de comportamento da curva de isolamento.

\subsection{Amostras com instalações}

A Figura 5 apresenta uma análise comparativa entre a amostra de referência e a amostra $\mathrm{S} 3$, com duas caixas de instalação elétrica inseridas na mesma face; e S4, com duas caixas de instalação inseridas de faces opostas.

Pode-se afirmar que as curvas das amostras com instalações elétricas (S3 e S4) apresentam poucas semelhanças com o isolamento da amostra de referência. Graficamente, pode-se observar uma variação relevante entre o isolamento da parede de referência e as amostras S3 e S4 para bandas de baixa frequência abaixo de $125 \mathrm{~Hz}$. 


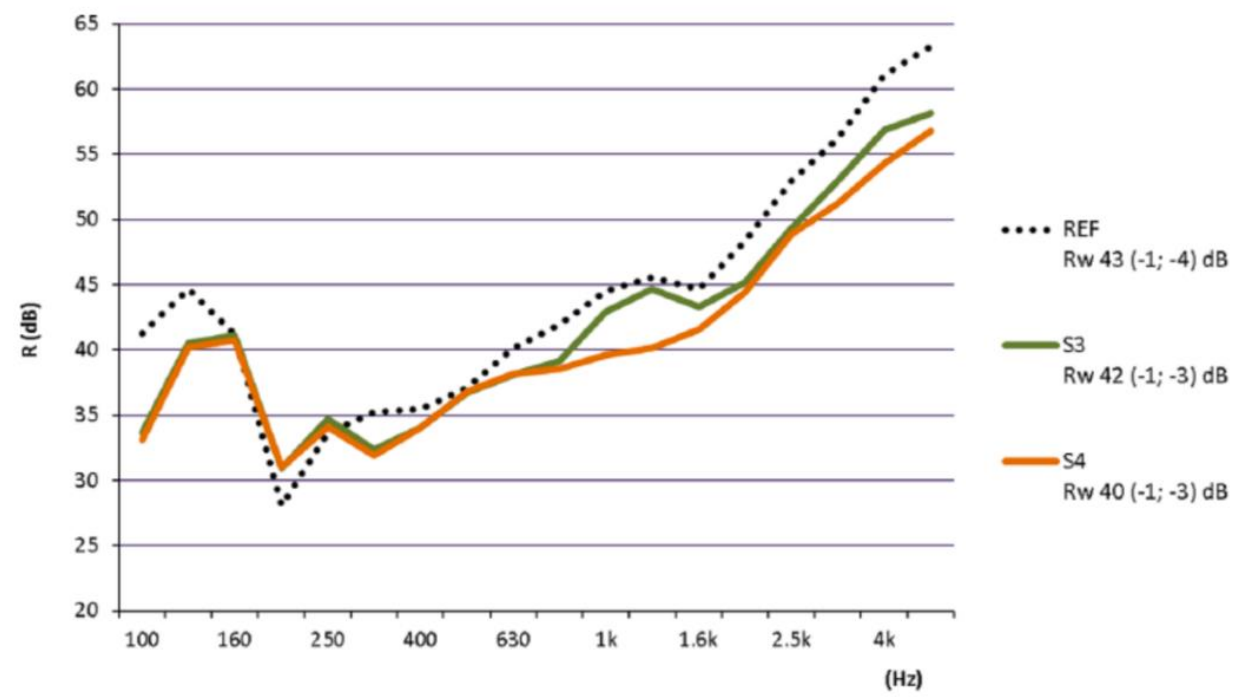

Figura 5 - Comparação entre os resultados da parede de referência REF e as amostras S3 e S4

No entanto, a frequência ressonante permaneceu entre 160 e $200 \mathrm{~Hz}$ para todas as paredes avaliadas, com reduções menos pronunciadas para S3 e S4. Novamente, uma segunda perda de isolamento foi observada entre as faixas de frequência de 250 e $315 \mathrm{~Hz}$.

Nas bandas de médias frequências, as amostras com caixas de instalação elétrica apresentaram comportamentos previsíveis para o isolamento acústico, uma vez que a perda de massa foi insignificante, mas a flutuação da curva ocorreu como resultado das aberturas na parede, sem perdas significativas na banda central de $500 \mathrm{~Hz}$. Em relação à amostra S3, com caixas apenas em sua face interna, foram verificadas as mesmas frequências críticas nas bandas entre 1250 e 1600 Hz. No entanto, a curva S4 não se comportou de maneira semelhante, com perdas expressivas de isolamento dentro do intervalo nas bandas de frequência de 800 a 1600 Hz, em comparação com a situação anterior.

Os resultados de Rw para as amostras S3 e S4 foram menores em 1 e 3 dB, respectivamente, em comparação com a amostra de referência. Isso indica uma redução de isolamento acústico do sistema de construção, principalmente quando projetos complementares preveem pontos de instalação elétrica alinhados para dois diferentes ambientes.

\subsection{Resumo dos resultados}

É evidente que o índice de redução sonora mostrou maior variação nas bandas de baixas frequências, principalmente para as amostras S3, S4 e S2, que tiveram aberturas consideráveis nos dois primeiros e múltiplas lacunas no último, modificando o comportamento do isolamento para sons de baixa frequência. Entretanto, as frequências ressonantes de todas as amostras ocorreram entre 160 e $200 \mathrm{~Hz}$, com exceção da amostra S2, que apresentou uma diminuição suave, possivelmente alterando sua ocorrência para frequências mais baixas.

Nas frequências médias, apenas a curva da amostra S4 não apresentou um comportamento típico, resultando em maior transmissão sonora, principalmente acima da frequência central de $630 \mathrm{~Hz}$, om aumento da frequência de coincidência comum a todas as paredes. Em relação à frequência crítica, ocorreu queda de isolamento entre as bandas de 1250 e $1600 \mathrm{~Hz}$, independentemente das estruturas medidas. A maior transmissão sonora das amostras, comparada à parede de referência, ocorreu nas bandas de altas frequências, fato explicado pelo menor comprimento de onda, propiciando uma propagação mais direta, ou seja, que se depara com menos obstáculos.

Pode-se afirmar que as perdas de isolamento acústico provocadas por falhas nos sistemas construtivos são de aproximadamente $2 \mathrm{~dB}$, assim como Craik e Evans e Mateus e Pereira (1989) observaram quando apontaram a mesma diferença de $2 \mathrm{~dB}$ para ensaios realizados em campo. A Figura 6 apresenta os valores de Rw obtidos para cada amostra. 


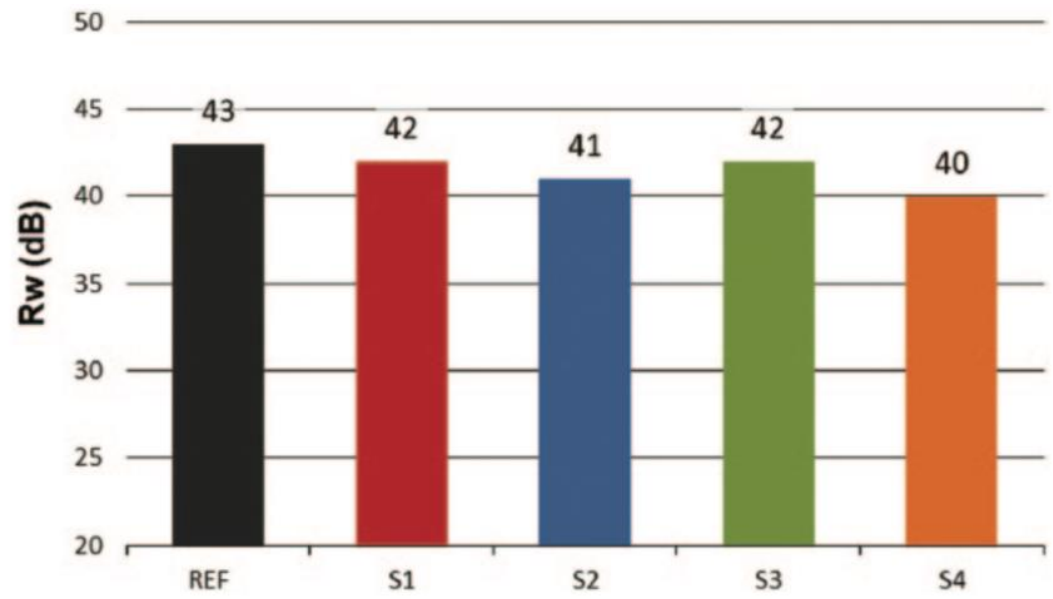

Figura 6 - Rw das amostras estudadas

\section{CONCLUSÃO}

Este artigo teve como objetivo avaliar a influência de falhas construtivas causadas durante a execução dos sistemas de paredes de alvenaria no desempenho acústico, com o objetivo de simular, em laboratório, situações que podem ocorrer no canteiro de obras. Nesse contexto, pode-se dizer que as medições permitiram uma avaliação mais confiável com base em detalhes que devem garantir a eficiência do produto final. Os testes mostraram evidências de perdas de isolamento para todas as amostras em que foram induzidas falhas construtivas, em comparação com a parede de referência, principalmente a amostra sem preenchimento de juntas verticais e com a instalação de quatro caixas de instalações elétricas.

Desta forma, as falhas construtivas, mesmo visualmente não identificáveis, podem ser detectadas através da análise de curvas de isolamento, considerando que a densidade deste sistema de construção está em conformidade com a lei de massa teórica. Portanto, uma parede de alvenaria que siga procedimentos adequados de construção deve apresentar uma curva configurada conforme o modelo teórico previsto, diferentemente das que apresentam falhas, que não atendem os princípios da massa no isolamento acústico do sistema. Assim, é aceitável caracterizar uma parede conhecida em relação a defeitos que possam estar afetando-a, através de uma série de medições que identificam o comportamento da curva de isolamento em todas as situações prováveis, em comparação com a referência. Considerando sempre o mesmo sistema construtivo, é possível identificar erros de construção em campo com mais eficiência.

\section{REFERÊNCIAS}

ASTM C67-17: 2017. Métodos de teste padrão para amostragem e teste de tijolo e telha estrutural de argila.

BALLOU, G. Manual para engenheiros de som. Oxford: Taylor e Francis, 2008.

BARRETT, K. Obra defeituosa. Reino Unido: Blackwell Publicação: John Wiley Sons, 2009.

CARRASCAL, T.; et al. Erros comuns e boas práticas em design e mão de obra. In: Birgit Rasmussen e Maria Machimbarrena. Ação COST TU0901 - Acústica de construção em toda a Europa. Volume 1: Rumo a um quadro comum na construção de acústica em toda a Europa (programa-quadro de IDT da UE), Viena, 2014, pp. 181-212.

CEN EN 1015-10: 1999. Métodos de ensaio para argamassa para alvenaria - parte 10: determinação de granéis secos densidade da argamassa endurecida.

CEN EN 1015-6: 1998. Métodos de ensaio de argamassa para alvenaria - parte 6: determinação da densidade aparente de argamassa fresca.

CRAIK, R.J.M.; EVANS, D.I. O efeito da obra na transmissão do som através de edifícios: parte 2 - Som com estruturas. Appl Acoust 1989; 27: 137-145.

DEL VAL L.; et al. Tradução entre descritores de isolamento acústico de impacto harmonizado existentes e propostos e alinhamento dentro de um esquema de classificação acústica comum proposto para edifícios. Appl Acoust 2018; 129: 204-216. 
DEMIRKALE, S.Y.; ASCIGIL-DINCER, M. Modernização de fachadas e fachadas de tijolos de cavidade para diferentes ruídos zonas usando medições de laboratório. Build Acoust 2017; 24: 77-100.

FAUSTI, P.; et al. Erros comuns durante a construção de novos edifícios e efeito de mão de obra. In: Anais do simpósio europeu - Harmonização dos descritores europeus de isolamento sonoro e padrões de classificação, Florença, 2010, http://www.acustica-aia.it/AIA_EAA_COST_FLORENCE_2010/pdf14 / EAA-COST_2010_07.pdf.

FRINGUELLINO, M.; SMITH, R.S. Transmissão de som através de paredes ocas de tijolo. Build Acoust 1999; 6: 211-224.

GOMPERTS, M.C; KIHLMAN, T. A perda de transmissão de som de aberturas circulares e em forma de fenda nas paredes. Acustica 1967; 18: 144-150.

HOPKINS, C. Isolamento acústico. Burlington, NJ: Elsevier, 2012.

ISO 10140-2: 2010. Acústica - medição laboratorial de isolamento acústico de elementos de construção - parte 2: medição do isolamento acústico no ar.

ISO 10140-4: 2010. Acústica - medição laboratorial de isolamento acústico de elementos de construção - parte 4: procedimentos e requisitos de medição.

IOSHIMOTO, E. Incidência de manifestações patológicas em edificações habitacionais. In: Tecnologia de edificações. p.545-548. São Paulo: PINI/IPT, 1988.

JONES, R.E. Como prever com precisão o isolamento acústico das partições. Sound Vib 1976; 10: 14-25.

KLIPPEL, F.S.; et al. Influência de diferentes espessuras de revestimento de argamassa no desempenho acústico de paredes. In: Anais do XII SBTA, 22-24 de agosto de 2017. São Paulo, Brasil: USP, ANTAC.

lajes. J Appl Phys 2010; 108: 064903.

KLIPPEL, F.S.; et al. Influência da espessura de revestimentos de argamassa no desempenho acústico de alvenarias de blocos cerâmicos. Ambiente Construído, v. 19, n. 2, p. 145-156. Porto Alegre, $2019 . \quad$ Doi: http://dx.doi.org/10.1590/s1678-86212019000200313.

MATEUS, D.M.R.; PEREIRA, A.S.C. Influência de pequenos erros de execução em obra sem desempenho acústico de edifícios: exemplos típicos. In: Anais da TecniAcustica, Cáceres, 2011. Sociedad Española de Acústica, http://www.sea-acustica.es/fileadmin/Caceres11/Caceres11_AED005.pdf.

MESOMO, M. F. Manifestações patológicas em unidades habitacionais construídas com paredes de concreto moldadas in loco com fôrmas metálicas: análise das falhas observadas na etapa de execução. Trabalho de conclusão de curso. Universidade Federal do Rio Grande do Sul. Porto Alegre, 2018.

NICHELE, B. P. Manifestações patológicas em unidades habitacionais construídas com paredes de concreto moldadas in loco com fôrmas metálicas: análise das falhas observadas na etapa de execução. Trabalho de conclusão de curso. Universidade Federal do Rio Grande do Sul. Porto Alegre, 2014.

PETERS, R.J.; SMITH, B.J.; HOLLINS, M. Acústica e controle de ruído. 3rd ed. Nova York: Taylor \& Francis, 2013. SOUSA, M.W.S.; CABRAL, A.E.B. Levantamentos de erros executivos em edifícios em alvenaria estrutural de bloco cerâmico. XV ENTAC 2014; 1015-1024, http://www.infohab.org.br/entac2014/artigos/paper_22. pdf.

SUGIE, S.; YOSHIMURA, J.; IWASE, T. Efeito da inserção de um ressonador Helmholtz no isolamento acústico de um cavidade de partição de duas folhas. Acoust Sci Technol 2009; 30: 317-326.

WANG, X. Teoria da transmissão sonora ressonante através de pequenas aberturas em perfurações periódicas. $J$ Appl Phys, 2010; 108: 064903. 